\title{
Assessing the Impact of Real-Time Occupancy State Transitions on Building Heating/Cooling Loads
}

\author{
Zheng Yang, Burcin Becerik-Gerber \\ Department of Civil and Environmental Engineering, University of Southern California, 3620 S. Vermont \\ Avenue, Los Angeles, California, United States
}

\begin{abstract}
Even though it has been widely accepted that occupancy is one of the most important factors impacting energy use of HVAC systems, how occupancy is associated with heating/cooling loads for sustained and maximum energy efficiency in multi-zone buildings is still not well understood. This study analyzes the influence of occupancy on heating/cooling loads at the building level using occupancy state transitions. A data-driven approach is developed using an enhanced variable neighborhood search algorithm to determine setpoint controls for individual zones and to minimize the heating/cooling loads based on occupancy state transitions. Simulations are used to compare the loads after implementing the proposed approach with the baseline control to understand the relationships. Based on the results, the optimal combinations of setpoint/setback schedules and distances for each zone were identified to minimize heating/cooling loads at the building level. The convergence of the search was not influenced by different occupancy assignments or initial solutions, and there was no random solution that could outperform the proposed approach to reduce heating/cooling loads based on occupancy transitions. A minimum of $10.4 \%$ and a maximum of $28.3 \%$ load reduction were achieved in the case study building, compared to the baseline control.
\end{abstract}

Keywords: Occupancy; State transition; Heating and cooling; Loads; Setpoint Control; Energy Efficiency; Simulation

\section{Introduction}

Buildings account for approximately $40 \%$ of the energy consumption in the United States. $46.8 \%$ of this amount is attributed to commercial buildings [1]. In commercial buildings, more than $40 \%$ of the energy is consumed by the HVAC (heating, ventilation, and air conditioning) systems to maintain desirable and healthy thermal conditions [2,3]. However, it is estimated that $90 \%$ of the building HVAC systems are not energy-efficient to some extent [4]. There is a significant difference between the energy required for actual heating/cooling needs in buildings and the energy consumed by these systems. To increase HVAC system energy efficiency, one strategy is to improve a building's physical characteristics, material thermal properties, and to replace old building systems with newer ones. However, implementing these solutions in existing buildings might not be feasible due to lack of available financial and human resources. An alternative way to achieve energy efficiency is to operate HVAC systems for solely meeting actual demands. It has been widely accepted that occupants of buildings are one of the most important factors to determine the actual demands for heating and cooling [5-8]. Therefore, understanding actual heating/cooling demands based on occupants is an effective solution to improve HVAC system energy efficiency without sacrificing occupant comfort and system functionality. In general, the importance of building occupants can be broken down into two categories: occupancy, which represents when (presence) 
and how many (number) persons occupy a building; and actions, which are about how occupants interact with building elements, such as windows and blinds. Since occupancy is the basis for activities and closely associated with interior heat gains (human metabolism, as well as use of appliances/equipment) and conditioning requirements, there is potential for energy efficiency if occupancy is considered in order to match the operations of HVAC systems with actual heating/cooling demands.

Even though extensive research has been conducted to improve HVAC system energy efficiency by responding to actual occupancy, the significance and form of influences that building occupancy put onto heating/cooling loads are still not well understood. It is also not well-defined how and when occupancy should be integrated with heating/cooling control for sustained and maximum energy efficiency. Occupancy in buildings has patterns but is stochastic in nature and has variant state transitions. Occupancy state transitions (short as "occupancy transitions" here thereafter) are defined as the switches between different occupancy states (e.g., arrival, departure, and intermittent absence). Linking occupancy with actual heating/cooling loads for an HVAC system is a complex problem as there exist load exchanges through heat transfer and balance across different zones. This paper introduces a data-driven approach to understand the relationships between occupancy transitions and heating/cooling loads for multi-zone buildings. An Enhanced Variable Neighborhood Search (EVNS) algorithm is introduced to determine the setpoint control for each zone, by which the minimum heating/cooling loads at the building level could be estimated based on occupancy transitions. Given the occupancy data and weather, the differences in heating/cooling loads are calculated by the algorithm introduced in this paper. The corresponding loads are then compared with the baseline control to understand the relationships between occupancy transitions and heating/cooling loads.

The paper is structured as follows. In Section 2, we introduce the importance of integrating occupancy transitions with heating/cooling loads analysis and review the existing studies of occupancy transitions based setpoint control and discuss the gaps, and summarize the objectives of our research. In Section 3, we introduce the approach for the Enhanced Variable Neighborhood Search to determine setpoint control for individual zones for enabling building level energy efficiency. We provide the validation results in Section 4, using a small size reference building. Finally, in Section 5, we present the results for finding the relationships between occupancy transitions and heating/cooling loads using a real-world case study for our occupancy-loads analysis. In Section 6, we conclude the paper and discuss the limitations and future work.

\section{Importance of Occupancy State Transitions}

\subsection{Occupancy State Transitions and Loads}

For building HVAC systems, the amount of heating/cooling loads depends on the level of thermal condition that is required. As introduced earlier, occupancy transitions are the switches between different occupancy states, thus not all of the loads are effective for heating/cooling when the zone is not occupied. Since many of the HVAC systems respond to heating/cooling loads through the control of a setpoint and the majority of office buildings in the United States have been equipped with thermostat for setpoint control as of 2011 [9], setpoint is used in this paper as the medium to manipulate the interactions among occupancy, loads, and thermal conditions. During an unoccupied period, allowing the setpoint to float to a different temperature, which is defined as the setback (higher than the setpoint when cooling is required, and lower than the setpoint when heating is required), could potentially reduce heating/cooling loads and 
improve an HVAC system's energy efficiency. Generally, heating/cooling loads of a setpoint control can be divided into four periods based on occupancy transitions (Figure 1): (1) Setpoint period: heating/cooling is provided by the terminals for zones to maintain the temperature within the range of the setpoint, and heating/cooling loads are considered as effective ("effective" is defined in this paper as actual demand for the HVAC system); (2) Float period: heating/cooling is not provided for the zones and the temperature is allowed to float from setpoint to setback, and heating/cooling loads are considered not effective and only minimum air flow is supplied per the ASHRAE requirement; (3) Setback period: heating/cooling is provided by the terminals to prevent room temperature from exceeding the setback, and heating/cooling loads are considered effective. To be clear, setback period does not mean the setback must be maintained. Setback acts as a threshold to keep the temperature within the range between the setpoint and setback; (4) Reconditioning period: heating/cooling is provided for the zones to restore the temperature from setback or the floating point to setpoint, and heating/cooling loads are considered effective. Therefore, the relationship between occupancy transitions and heating/cooling loads could be reformulated as the relationship between the transitions of occupied/unoccupied states and the transitions of loads that are actual demands for heating/cooling versus the loads that do not need to be addressed by an HVAC system (Figure 1).

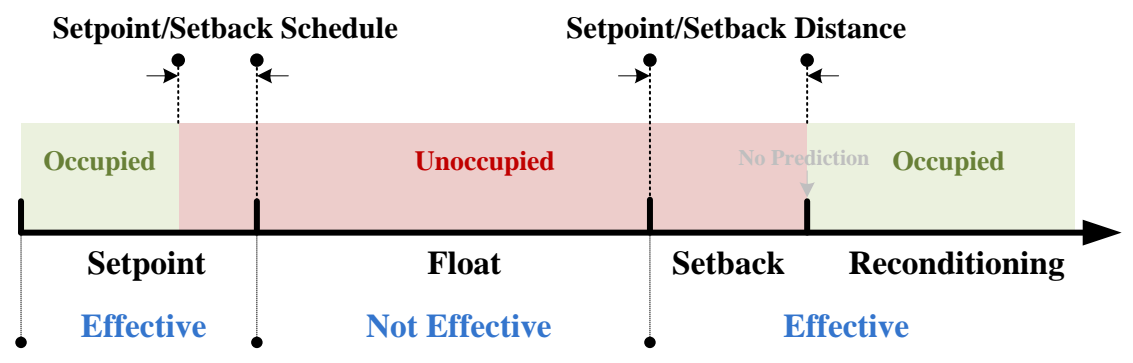

Figure 1: Relationship between occupancy transitions and heating/cooling load transitions

Conceptually, the waiting time to start the float period after a zone becomes unoccupied (defined as the setpoint/setback schedule), and the absolute value of setback minus setpoint (defined as setpoint/setback distance) to start the setback period determine the deviations between occupancy transitions and load transitions. Different combinations of setpoint/setback schedules and distances may lead to different levels of energy efficiencies. For example, to demonstrate this point, we simulated a real-world office building (the same building is also used in this paper - see Section 5) in a study [10], where we used 73F as the static setpoint (based on the ASHRAE comfort compliance and the Department of Energy reference buildings' default settings), when the zone was occupied. The interval for setpoint/setback distance was 1 $\mathrm{K}$ and the interval for setpoint/setback schedule was 5 minutes. The conditioning miss and energy reduction for the third floor of the building were simulated for a year from March 2014 to March 2015. Conditioning miss was defined as the length of time when the space is occupied but the temperature is outside the comfort range (it was defined as $73 \mathrm{~F}$ with $\pm 1 \mathrm{~K}$ deadband). Thermal comfort was not within the scope of this paper but these parameters and assumptions about thermal comfort were made for demonstrating the effectiveness of the proposed method. There might be other setpoints and comfort ranges for different occupants. If this information is available, it can be used as an input to replace $73 \mathrm{~F}$ and $73 \mathrm{~F} \pm 1 \mathrm{~K}$. Energy reduction was calculated as the absolute amount of energy savings. The results are visualized (Figure 2) using gray maps (percentage of energy reduction and percentage of conditioning miss are compared to the baseline control, which maintains setpoint of $73 \mathrm{~F}$ for the working hours of 

energy reduction and less conditioning miss were achieved.
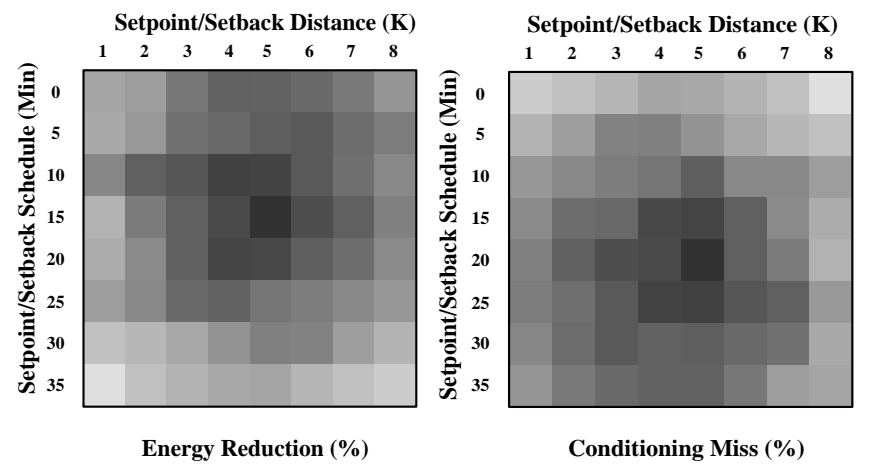

Figure 2: Energy implication of different combinations of setpoint/setback schedules and distances

Within the context of the comfort parameters chosen, the simulated results demonstrate that setpoint/setback schedule and distance are important to reduce heating/cooling energy use while maintaining occupant comfort. Generally, when a zone becomes unoccupied, instant control adjustments may cause discomfort as an occupant may reoccupy his/her office soon after leaving it. Sometimes reconditioning after a relatively short period of vacancy may consume more energy than just maintaining the setpoint. Especially when a setback is far from a setpoint, the transient large amount of loads could result in additional energy consumption and time to recover from the setback. We demonstrated the transitions of loads do not necessarily follow the transitions between occupied/unoccupied states by using the different combination analyses in [11]. A portion of the loads during unoccupied periods should be considered effective for improving energy efficiency (Figure 1). The boundaries of the portions are represented by the setpoint/setback schedules and distances, and determine the relationships between occupancy transitions and heating/cooling loads. Since errors might be introduced when prediction is used to pre-recondition the space prior to an occupant occupies his/her office, the transitions between unoccupied/occupied states are considered the same as the transitions between setback and reconditioning periods (Figure 1) to eliminate uncertainty of the analysis for occupancy transitions-loads relationship.

\subsection{Occupancy State Transitions and Heating/Cooling Control}

Several research initiatives have been undertaken to optimize HVAC system setpoint controls based on occupancy transitions [12,13]. The basic principle is that energy efficiency could be improved by not fully maintaining static setpoints in vacant zones and only providing minimum airflow per the standards/codes [14]. Substantial energy savings have been reported by prior research [15-17] by allowing zone temperatures to float within a certain range, depending on whether a zone is occupied or not [18], and setpoint/setback switch schedules were optimized between occupied and vacant modes to reduce frequent system startups while maintaining occupant comfort [19]. For instance, the NEST thermostat is a self-programming thermostat that can create setback schedules by learning the changes occupants make to a setpoint, sensing the presence of occupants, and estimating the time required to reach the setpoint [20]. Smart Thermostat, an approach developed by Lu et al. [21], enables dynamic HVAC system on/off control based on Hidden Markov Chain for occupancy transitions. Through the use of simulations, the authors analyzed the energy savings achieved by not providing conditioning during unoccupied periods in 
eight homes and reported $28 \%$ of HVAC system energy savings. Another example is the Telkonet SmartEnergy control system [22], which allowed temperatures to vary within a relatively wide range in vacant residences. After occupants returned, the temperature was adjusted to its normal range within a lag time. The lag was determined based on tolerable recovery time set by occupants, residence type, HVAC type, and weather. However, these solutions are not scalable to commercial buildings with strong zone interdependencies. Erickson et al. designed the OBSERVE system, a temperature control algorithm, to adjust temperature based on occupancy in current time step and in the next hour [23]. Agarwal et al. proposed to set back the temperature setpoint in unoccupied zones and occupied zones. In a simulation, temperature was maintained at $73 \mathrm{~F}$ for occupied zones and $79 \mathrm{~F}$ for unoccupied zones. It was reported that $15 \%$ of the HVAC related energy consumption could be reduced [24]. Optimizing the switch time step between occupied and unoccupied modes, in order to avoid frequent system startups while maintaining comfort, has also been explored by previous work [19]. Although these research efforts considered the deviations of transitions between the setpoint control periods and the transitions between the occupancy states for energy efficiency, the analysis for the influence of occupancies in adjacent zones on heating/cooling loads was not considered. Personal Environment, a system developed by Johnson Controls, controlled the thermally connected spaces like cubicles to reduce the energy consumption by not conditioning unoccupied spaces [25]. RoomZoner was designed to change the conditioned air for each room based on occupancy and temperature [26]. Both of these studies focused on conditioning the parts of a building that are actually occupied, but lacked the ability to quantify how much energy could potentially be saved.

Different from the feedback-based mechanisms, which react only to occupancy and thermal condition changes, equation based setpoint optimizations, like Model Predictive Control (MPC) [27], are another direction to improve HVAC system energy efficiency based on occupancy transitions. In a study, where MPC was implemented, energy savings of $34 \%$ were reported for a typical summer day in Switzerland and $37 \%$ for a typical winter day in a city in the southeastern United States [28,29]. However, the design and implementations of such solutions are limited to specific HVAC systems and the performances highly depend on the selection of the system control parameters. Setpoint control could even increase energy usage by up to $10 \%$ if not programmed properly [18]. Other recently published studies for improving building energy efficiency focused on: impacts of building/system properties on selection of setpoints [30,31]; relationships between loads and occupant behavior variables in residential buildings and single zones [32-34]; integration of predetermined HVAC system settings with different occupancies [35-37]; and tradeoffs among building energy use, occupant comfort, and control complexity [38-40]. Yet, none of these studies has systematically investigated the influences of different occupancy state transitions among multiple zones on HVAC system energy efficiency at the building level.

In summary, the relationships between occupancy state transitions and heating/cooling loads for commercial buildings are not well defined. There generally exist load exchanges among zones through heat transfer and balance if there are temperature differences among the zones and openings (e.g., doors and windows) for ventilation [41]. Occupancy transition based setpoint control, for commercial buildings with multiple zones, requires the consideration of synergies among adjacent zones and global optimization of setpoint control for all zones [42]. Therefore, the objective of this study is to develop a data-driven approach to quantify the relationships between occupancy transitions and heating/cooling loads at the building level for HVAC system energy efficiency. The idea behind the presented approach is to search the optimal setpoint/setback schedules and distances for all individual zones to minimize 
heating/cooling loads for the entire building based on occupancy transitions, given the occupancy data and weather for a certain period of time. To be clear, the impact of different occupant numbers in each room is considered as a time-variant coefficient to the state of presence for adjusting heat gains. For example, two occupants in a space generate more heat than one occupant in the same space due to human metabolism and result in more cooling loads associated with heat generating appliances, such as computers. In this study, the increase and reduction of cooling loads are considered in the simulation for calculating the data-driven setpoint/setback schedules and distances of individual zones.

\section{Data-Driven Search Algorithm}

The goal of our data-driven approach is to minimize heating/cooling loads by searching the optimized setpoint control for each zone, based on the analysis of occupancy data and simulated energy data. The corresponding loads are then compared with the baseline control to understand the relationships between occupancy transitions and heating/cooling loads. To make the approach generalizable, we assumed that all of the zones have different combinations of setpoint/setback schedules and distances, and the scenario that all of the zones in a building share the same combination is considered as one possible solution among many. The synergies among zones are considered by the basic assumption of this data-driven approach that different zones requires different combinations of setpoint/setback schedules and distances to minimize the loads at the building level based on occupancy state transitions. For a certain period of time, synergies are analyzed in a model-free way through the search of optimal combinations for all zones in the building.

If there are $N$ zones and $T$ different combinations of setpoint/setback schedules and distances for each zone, $(T)^{N}$ possible solutions are in the solution space (N-dimensional) to simulate and evaluate. An exhaustive search is not feasible for large buildings when periodical (e.g., monthly or weekly) analysis is required, as the computational time and the number of simulations increase exponentially. For this combinatorial optimization problem, the algorithms that use heuristics have the potential to partially search the solution space and find the near-optimal solutions in a reasonable amount of time and within a reasonable computational power. In the building energy domain, for each solution, heating/cooling loads of the HVAC system have to be calculated through a simulation program or state-space thermal models, which are stand alone processes in addition to the optimization process and therefore time consuming. In addition, the loads of different solutions are discrete and independent, making the search speed and precision much lower than equation-based optimization problems, thus the performances of commonly used optimization techniques are expected to be lower. Specifically, simple local search like hill climbing in a greedy way may easily reach local optimums and stop searching [43]. Genetic algorithm is influenced by the selection of the parameters, such as the mutation and crossover rates, and is ineffective for local search and inefficient for precise search [44]. Simulated Annealing has little information about the search space and cannot target the subsets, which have higher chance to contain optimal solutions. It may further reduce the convergence speed [45]. Tabu Search could avoid the repeated search for local optimum. However, the performance of the search depends on the selection of the initial solution [46]. The speed of search, when Tabu Search is used, is also slow and not feasible to be used for the setpoint control optimization in multi-zone buildings. In order to solve these issues, we developed a data-driven algorithm called Enhanced Variable Neighborhood Search (EVNS) for determining the setpoint/setback schedules and distances for individual zones. The local search is first conducted in the neighborhood of solutions 
(combinations of setpoint/setback schedules and distances) and then the search is moved to other subsets of the solution space based on the heuristics (defined as the context information in this paper) of the present global optimums to avoid repeated search and the local optimum.

The EVNS is the integration of the Variable Neighborhood Search (VNS) algorithm and Particle Swarm Optimization (PSO), which we call the heuristic update in this paper. Although the VNS has been used for other applications, such as manufacturing equipment scheduling, to the best of our knowledge, this is the first time it is applied to determine setpoint/setback schedules and distances of connected zones in commercial buildings for achieving energy efficiency. Integration of the heuristic update with variable neighborhood search could increase the convergence speed and accuracy of the global optimum search. The first step is to conduct a local search of VNS in 1-neighborhood of the initial solution. The basic assumption of the VNS is that the local optimum under all possible neighborhood structures is considered as the global optimum. To be clear, one solution $x$ here is one set of combinations of setpoint/setback schedules and distances for all zones, and $X$ is the solution space for all possible solutions. $f(x)$ is the fitness of the solution, which is the heating/cooling loads of one set of combinations, calculated using energy simulation. The neighborhood structure is denoted as $N_{k}\left(k=1,2, \ldots, k_{\max }\right)$ and $N_{k}(x)$ is the set of all solutions in the $k$ - neighborhood of $x$. When there is no solution $\mathrm{x}^{\prime} \epsilon N_{k}(x) \subseteq \mathrm{X}$ that could make $f\left(x^{\prime}\right)<f(x)$, $x$ is considered as the local optimal solution. 1-neighborhood is defined as one step (either schedule or distance) from the $x$, 2-neighborhood is one step for both schedule and distance from the $x$, 3neighborhood is two steps for either schedule or distance from the $x$, and so on. A building with five zones is used as an example to illustrate the neighborhood structure (Figure 3). The black dots represent the setpoint/setback schedules and distances for individual zones with black lines showing the connections among these zones. The blue dots represent the 1-neighborhood of the current solutions of all zones while the red dots represent the 2-neighorhood. The red lines and blues lines show the paths of search. If k-neighborhood of $x$ is outside the boundaries of possible schedules or distances, it stays on the boundaries.

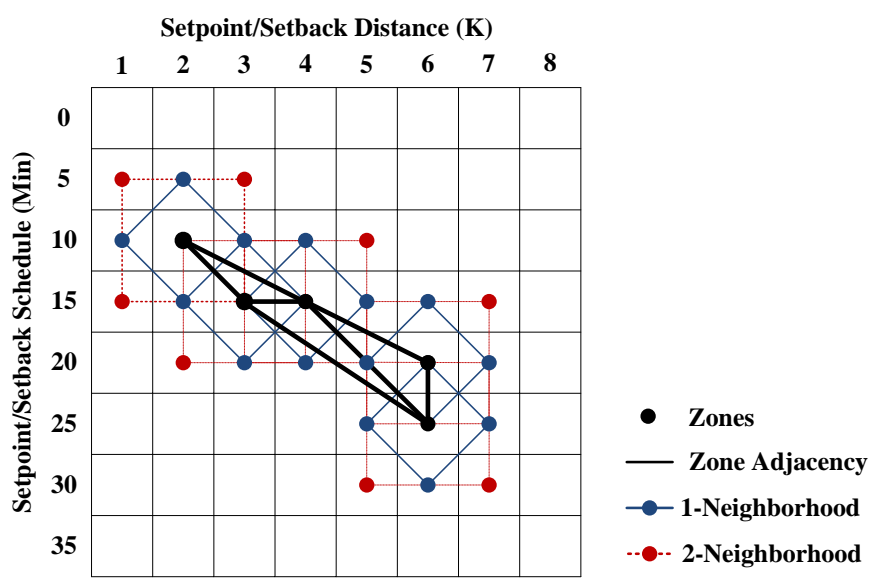

Figure 3: Neighborhood of solutions for the small reference building with five zones

In this paper, only 1-neighborhood is considered for the local search to reduce the time, complexity, and the possibility of missing the optimum, however it significantly increases the workload of the local search for global optimum as it is one smaller neighborhood at a time and is easy to end up in a random search as the properties of the solution space are unknown. In order to guide the search to other subsets of the 
solution space, a number of solutions in the neighborhoods of the local optimum is selected for the heuristic update until an optimal solution is obtained. To conduct the heuristic update, $m$ solutions are generated simultaneously and updated towards the current global optimums based on the amount of heating/cooling loads. Specifically, the combination of setpoint/setback schedules and distances for each zone is updated towards both the combination with the minimum zone level loads of the same zone and the combination in the solution with the minimum building level loads (Figure 4). The ith $(i \leqq m)$ solution can be denoted as $X_{i}=\left(x_{i 1}, x_{i 2}, \ldots, x_{i N}\right)$ to indicate the combination for each zone and its location in the solution space. The solution consisting of the best combinations for each zone with the minimum zone level loads is expressed as $Z_{i}=\left(z_{i l}, z_{i 2}, \ldots, z_{i N}\right)$, short as $z$ best. The solution consisting of the combinations with the minimum building level loads is expressed as $B_{i}=\left(b_{i 1}, b_{i 2}, \ldots, b_{i N}\right)$, short as bbest. In each iteration, the setpoint/setback schedules and distances for the $k t h$ zone $(1 \leqq k \leqq N)$ of the ith solution is updated based on the equation:

$$
\mathrm{x}_{i k+1}=a x_{i k}+c_{1} \operatorname{rand}()\left(\text { zbest }_{i k}-x_{i k}\right)+c_{2} \operatorname{rand}()\left(\text { bbest }_{i k}-x_{i k}\right)
$$

In which the $a, c_{1}$, and $c_{2}$ are non-negative constants to adjust the step length towards the zbest and bbest, and $\operatorname{rand}()$ is a random number in $[0,1]$.
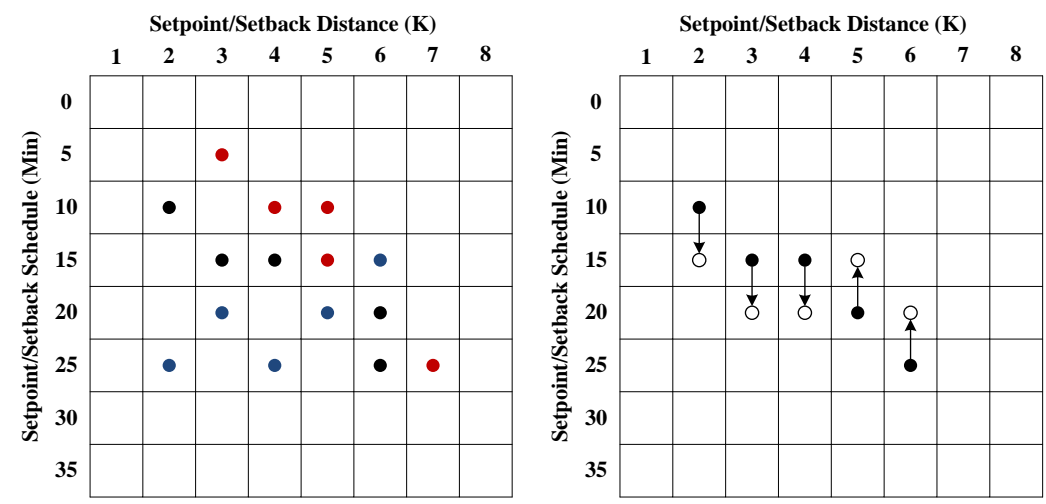

- Combination before update

○ Combination after update

- Best combination at zone level

- Best combination at building level

Figure 4: Heuristics for solutions to update

Finally, the two optimums are compared. If the new optimum is better than the local optimum, the entire process is iterated for the new optimum; otherwise the neighborhood is varied for generating new solutions to repeat the heuristic update. The detailed steps for one iteration are set as follows: 
Step 1: The initial solution $x$ is set as the best combination shared by all zones (all zones have same combinations of setpoint/setback schedules and distances). The neighborhood structure is set as $N_{k}$ $\left(k=1,2, \ldots, k_{\max }\right) . k_{\min }$ and $k_{\max }$ are set as the minimum and maximum neighborhood, respectively, $k_{\text {step }}$ is the step length of varying neighborhood for each iteration, and $t_{\max }$ is the maximum computation time;

Step 2: Search local optimum $x_{\text {best }}$ in the 1-neighborhood of $x$ and consider it as the current optimal solution:

Step 2-1: Step 1: Select an initial solution $x^{0}$ and set it as current optimal solution: $x^{*} \leftarrow x^{0}$, $T=N_{k}\left(x^{*}\right), k \leftarrow 1$;

Step 2-2: Select a solution set $S(S=(T+1) / 2)$ from $T$ and get the optimal solution $x^{+}$until $T \backslash\left\{x^{*}\right\}=\emptyset$. If $f\left(x^{+}\right)<f\left(x^{*}\right), x^{*} \leftarrow x^{+}$, and $T=N\left(x^{*}\right)$, otherwise $T \leftarrow T S$;

Step 2-3: Repeat Step 2 until all the solutions in the neighborhood is searched or the maximum time (including simulation time) is reached, then $k \leftarrow k+1$;

Step 3: Set $k=k_{\text {min }}$;

Step 4: Randomly select $m$ solutions $x_{i}^{\prime}(i=1,2, \ldots, m)$ in the $k$-neighborhood of $x^{\prime} \in N_{k}\left(x_{\text {best }}\right)$, and implement heuristic update to find the optimal solution $x$ ':

Step 4-1: Calculate the loads for each of the $m$ solutions based on the energy simulation;

Step 4-2: Compare the loads of each combination in each solution with zbest, if its loads are lower, the combination in the zbest is replaced by the combination in this solution;

Step 4-3: Analyze the trajectory of the heuristic update for each solution and compare the best one with the bbest, if better, the bbest is replaced by the solution;

Step 4-4: Calculate the update of each solution based on zbest and bbest;

Step 4-5: If the loads after the heuristic update reach the preset load level (e.g. 15\% load reduction), stop the update, otherwise go back to Step 1 until maximum iteration time (e.g., 100 times) is reached;

Step 5: Use $x$ ' as the initial solution and search its 1-neighborhood to find the local optimum x". If $f(x ")<f(x)$, set $x=x$ " and go back to Step 3, otherwise make $k=k+k_{\text {step }}$ and go back to Step 4;

Step 6: Iterate the above steps until no better solution could be found or the $t_{\text {max }}$ is reached.

\section{4. Algorithm Validation and Results}

3 A small reference building was used for validating whether the proposed approach could determine setpoint/setback schedules and distances for all individual zones to minimize heating/cooling loads based on the occupancy transitions, using EnergyPlus for computing the energy consumption and thermal conditions. We used the small size reference office building constructed after 2004 (Figure 5) [47]. Reference buildings are developed by the Department of Energy and National Energy Research Laboratories as standard building energy models, which represent $70 \%$ of the commercial building area in the United States. The location of the small size reference office building is assumed to be near LAX (Los Angeles International Airport) where the weather data are collected. Occupancy information was obtained from a real world office building (also used in the transitions-loads analysis in Section 5) using a cross- 
space occupancy modeling algorithm developed in [48]. Occupied and unoccupied states of occupancy in each room were calculated every minute. Generally, zones consist of more than one room and zone level occupancy transitions are obtained by aggregating the occupancy state of each room in that zone: if at least one room in a zone is occupied, the zone is occupied; if all rooms are vacant, the zone is unoccupied. The schedules of appliances and equipment were assumed to follow the occupancy statuses. In other words, if a space was occupied, equipment/appliances were assumed to be on and if a space was unoccupied, they were assumed to be off. Since there are five zones in the reference building, five occupants were randomly selected from the occupancy information and assigned to the reference building. The transitions of occupied/unoccupied states were then extracted and used to find the optimized setpoint control for each zone.

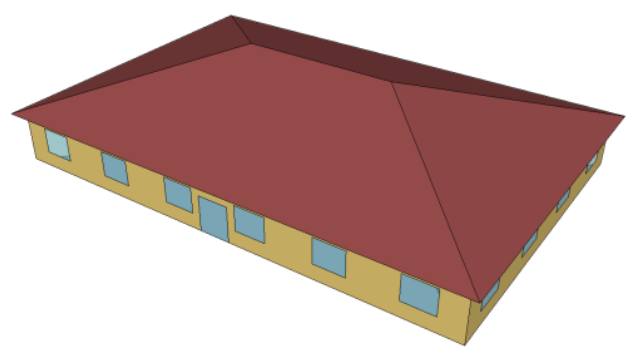

Figure 5: Small size reference office building

During the on-hour period (6:30AM - 9:30PM on workdays, and 7:00AM - 9:30PM on weekends), $73 \mathrm{~F}$ was used as the static setpoint to maintain a comfortable thermal environment when the zone was occupied and $73 \mathrm{~F}$ with $\pm 1 \mathrm{~K}$ deadband was assumed to be the comfort range. It is important to note that any other comfort range could be chosen or this comfort information could be an input to the algorithm from other comfort solutions. When the zone was vacant, the temperature was allowed to float until it reached the setback before the zone was occupied again. 8 different setback values (with $1 \mathrm{~K}$ as the interval from $74 \mathrm{~F}$ to $81 \mathrm{~F}$ ) for setpoint/setback distance and 8 waiting times (with 5 min as the interval) for setpoint/setback schedules were used to create 64 combinations. During the off-hour period, no cooling or heating services were provided. Only the minimum airflow was maintained to satisfy the ASHRAE compliance [49]. The EVNS was implemented in Matlab, by which the output of the simulation was set as input to the search and the output of the search became the input of the simulation. For each solution in each iteration, the period from March 2014 to March 2015 was simulated to output the heating/cooling loads.

The performance of the EVNS was evaluated by analyzing the convergence of maximum heating/cooling load reduction from the iterations. As a proof of concept, the entire process was repeated independently for 5 times (trials) with different occupants being randomly selected (among 28 possible occupants) and assigned to the reference building's zones (Figure 6). The initial condition for this analysis was based on the setpoint/setback schedule of 15 minutes and setpoint/setback distance of $5 \mathrm{~K}$ for all five zones (73 F for setpoint; $78 \mathrm{~F}$ for setback). 


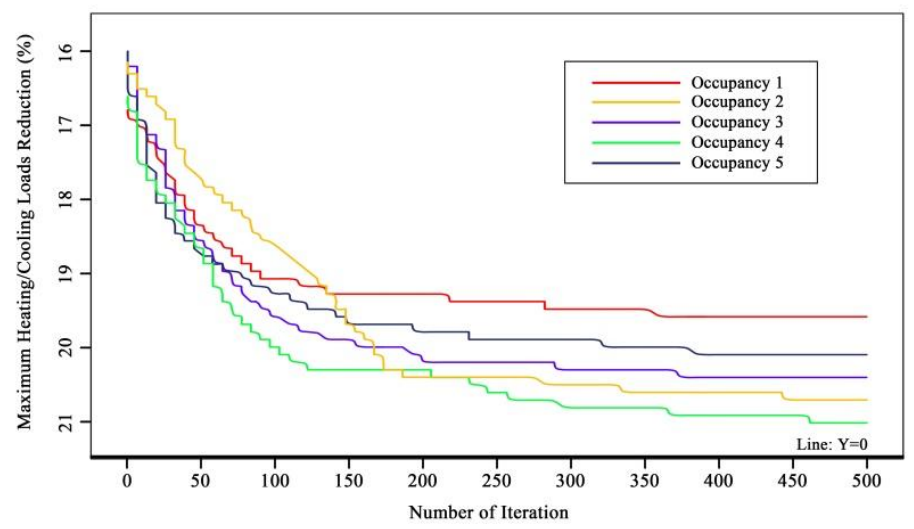

Figure 6: Trace progress of the maximum heating/cooling load reduction in 5 independent trials of search with different occupancy assignments

Based on the results of plotting the maximum heating/cooling loads for all iterations, the optimal combinations of setpoint/setback schedules and distances for each zone were identified to minimize heating/cooling loads at the building level. Although the difference in occupancy transitions (resulting from different occupancy assignments) influences the speed of convergence, the global optimum (solution with the global maximum heating/cooling load reduction) was found within 470 iterations. As a result, the number of required simulations is easier to finish within a reasonable time and computational power. To further investigate how initial solution affects the search performance, different initial solutions ( 5 trials) other than the shared combination for all zones were tested and the results were plotted in Figure 7. The initial solutions for this analysis were five random combinations of setpoint/setback schedules and setpoint/setback distances for the five zones (including $15 \mathrm{mins} / 5 \mathrm{~K}, 10 \mathrm{mins} / 7 \mathrm{~K}, 5 \mathrm{mins} / 7 \mathrm{~K}, 20 \mathrm{mins} / 3$ $\mathrm{K}$, and $10 \mathrm{mins} / 4 \mathrm{~K})$. The occupancy information for all five trials was the same. It can be seen that the selection of initial solution does not significantly influence the convergence speed and the level of maximum heating/cooling load reduction based on occupancy transitions remains the same. The trajectories of search in the solution space were different for different initial solutions, but they all approached the neighborhood of the global optimum around 200 iterations.

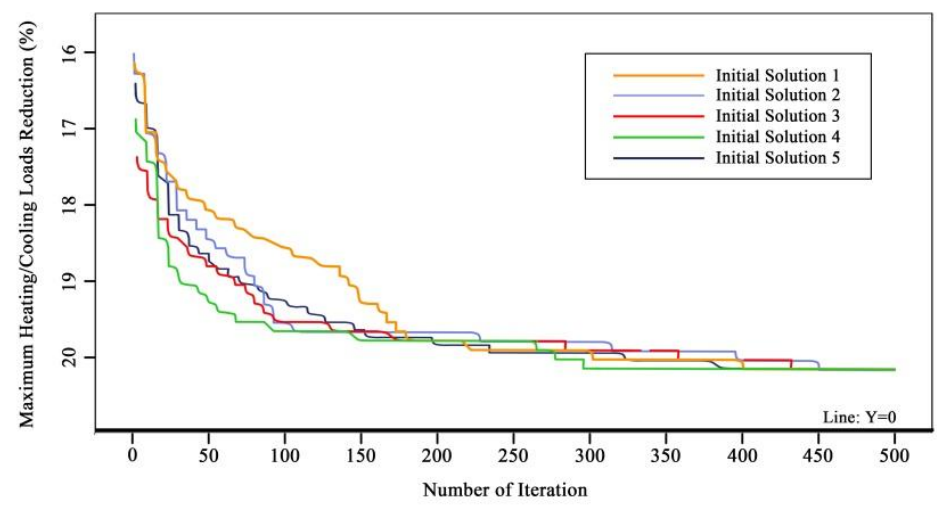

Figure 7: Trace progress of the maximum heating/cooling load reduction in 5 independent trials of search 
Lastly, the performance of the EVNS was compared with that of randomly formed combinations of setpoint/setback schedules and distances (defined as random solutions) for different zones to examine whether the solution from the EVNS could be considered as the global optimum or if it is only a local optimum. 500 solutions were generated and the corresponding heating/cooling loads were compared with the loads after implementing the EVNS (Figure 8). The results were presented as the statistics of percentages in terms of the load difference (histogram, density, and boxplot in Figure 8) between the random solutions and the solution from the EVNS, which is denoted as the line $\mathrm{Y}=0$. Random combinations resulted in the increase of heating/cooling loads from $0.2 \%$ to $25.8 \%$ (according to the boxplot). The difference was approximately Gaussian distributed (according to the Q-Q Plot) with the mean of $11.8 \%$ and the standard deviation of $5.1 \%$ (histogram and density plots), indicating the majority of the solutions had the similar ability to reduce certain amount of loads by not providing heating and cooling during some of the unoccupied periods. No randomly generated solution outperformed the solution generated by the EVNS for reducing the heating/cooling loads at the building level.
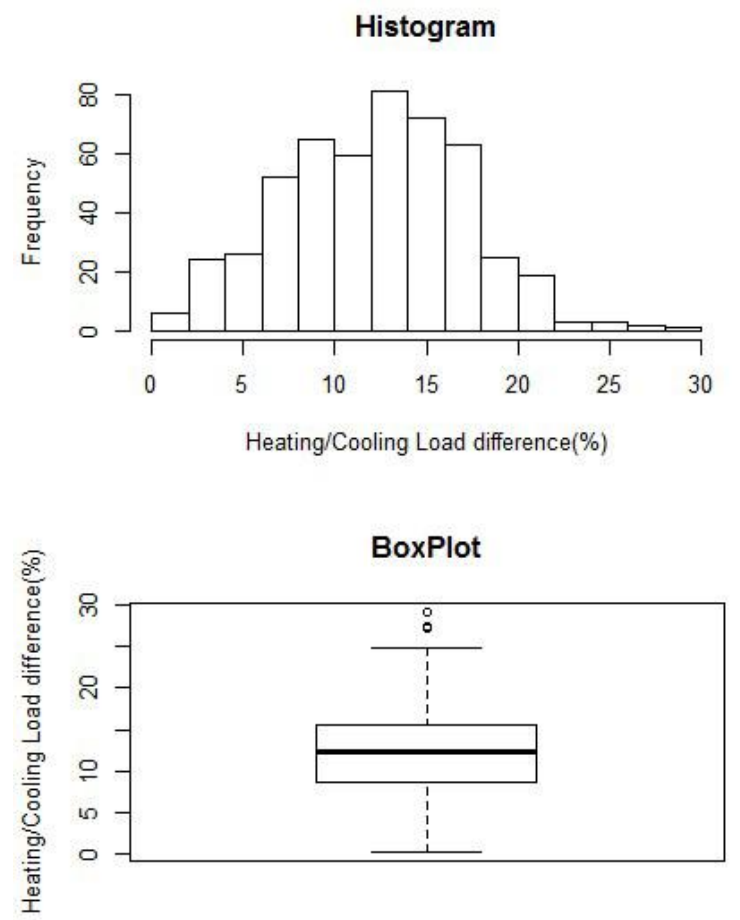
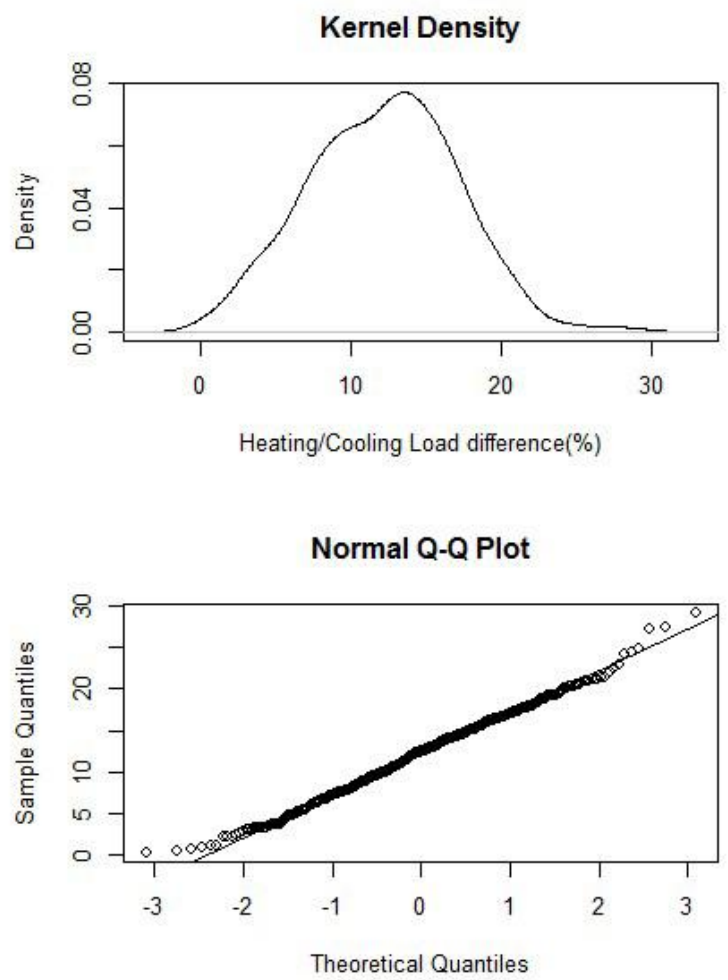

15

Figure 8: Statistics for load differences between implementing the EVNS and random combinations in the reference building

In summary, it is demonstrated that the EVNS is effective to determine the optimal combinations of setpoint/setback schedules and distances for different zones based on the occupancy transitions. The maximum heating/cooling load reduction could be achieved at the building level, the amount of which represents the relationship between the occupancy transitions and heating/cooling loads for a certain period of time. Energy efficiency (energy reduction and conditioning miss) for a specific HVAC system 
(e.g., HVAC VAV system) could be then improved by integrating the setpoint/setback control with

2 specific terminal control parameters. \\ 5. Transitions-Loads Analysis and Results}

The EVNS was then implemented in a simulation using the real-world office building as a case study to quantify the relationships between occupancy transitions and heating/cooling loads at the building level. In other words, we performed an occupancy-loads analysis to determine the setpoint control for all individual zones that could minimize heating/cooling loads based on occupancy transitions. The simulated building is a typical office building facing north on the University of Southern California (USC) campus in Los Angeles, California (Figure 9). It is a three-story building with 89 mechanically ventilated rooms that have spaces of varying sizes and functions. Most of the rooms in the building are enclosed single occupancy offices. Other rooms are classrooms, conference rooms, and auditoriums. The building is served by a centrally controlled HVAC system with variable air volume (VAV) boxes in thermal zones. Although the location of the building is 13 miles from LAX, this building is also assumed to be near LAX and the same weather data as in the first simulation case were used.

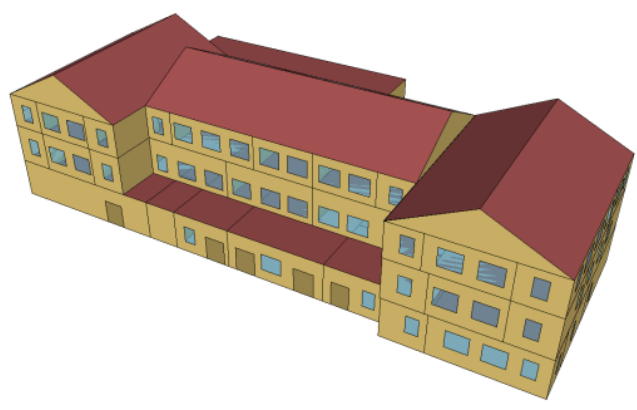

Figure 9: Real-world office building

The EVNS was used to search the optimal combinations of setpoint/setback schedules and distances for all 16 zones ( 28 rooms) on the third floor of the building. Since the EVNS could be used to quantify the occupancy transitions-loads relationship for any period of time, monthly analysis was conducted in this case study to be different from the reference building. The period of one year from March 2014 to March 2015 was divided into 12 months. For each month, the EVNS was run independently with the actual occupancy information (from the cross-space occupancy modeling algorithm). Same as the reference building, during the on-hour period (6:30AM - 9:30PM on workdays, and 7:00AM - 9:30PM on weekends), a setpoint ( $73 \mathrm{~F}$ ) was maintained and allowed to float until reaching a setback when the zone was unoccupied for more than certain minutes, and intermittently went back to the setpoint if the space became occupied again. During the off-hour period, no cooling or heating services were provided. Only minimum airflow was maintained to satisfy the ASHRAE compliance [49]. Again, 8 different setback values (with $1 \mathrm{~K}$ as the interval) for the setpoint/setback distance and 8 waiting time (with 5 min as the interval) for the setpoint/setback schedule were combined. An example of the combinations for all of the zones during one month (e.g., April) was shown in Figure 10. All the zones are numbered in a clockwise direction. For each zone, the red text shows the setpoint/setback schedule while the blue text shows the setpoint/setback distance, both determined by the proposed data-driven approach. 


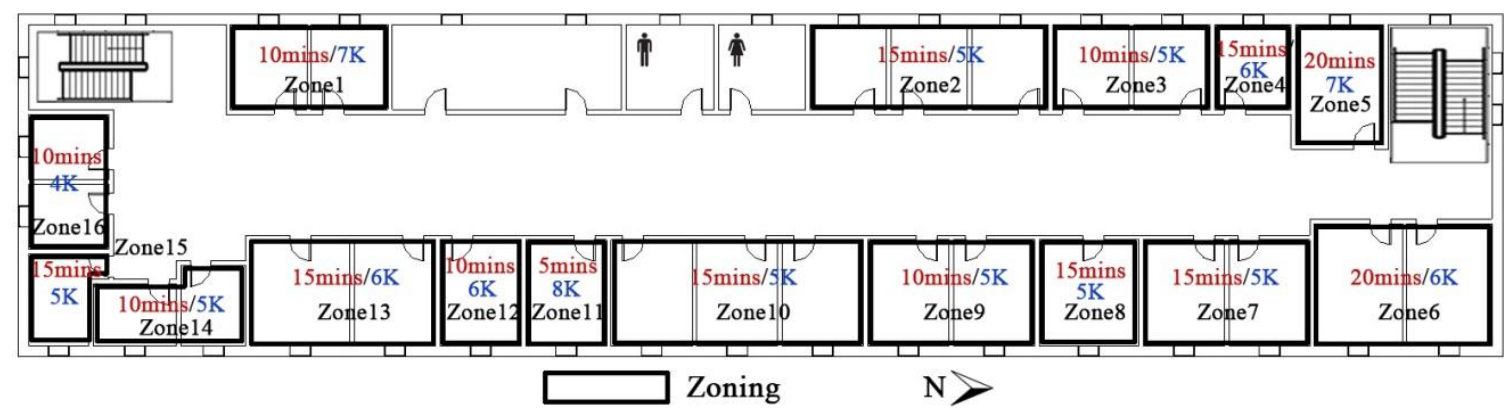

1

2 3

5

6

7

Figure 10: Setpoint/setback schedules and distances of all zones in the real-world building for the month of April

The heating/cooling loads after implementing the EVNS were compared with the loads under baseline control. The results are shown in Figure 11, in which the performance of baseline control is denoted as the line $\mathrm{Y}=0$.

Monthly Load Difference

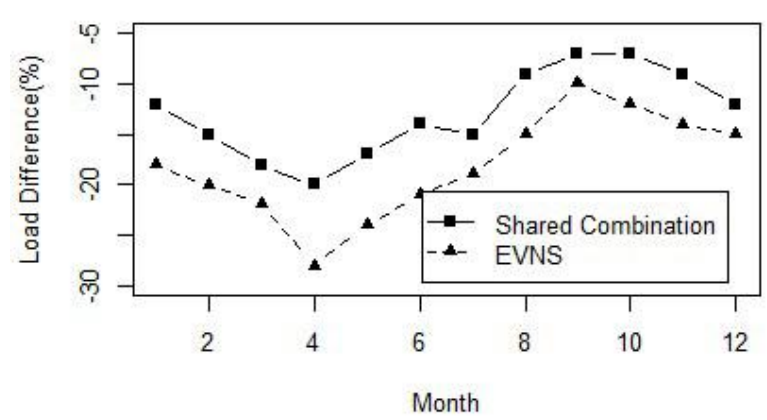

\section{Correlation}

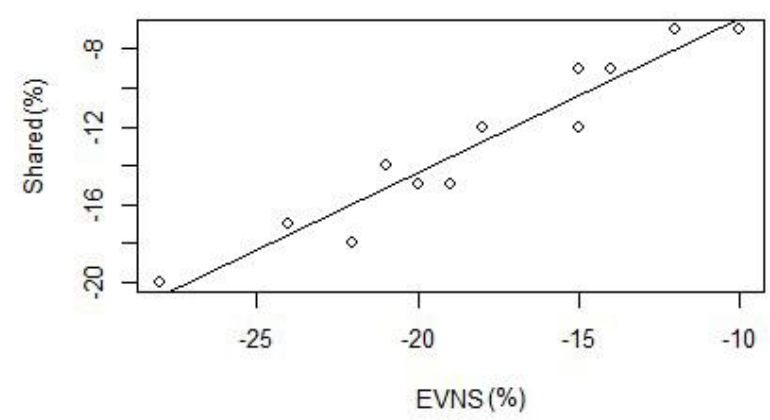

BoxPlot

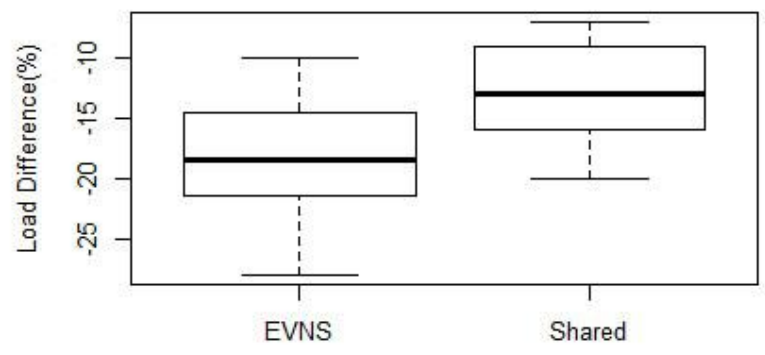

Kernel Density

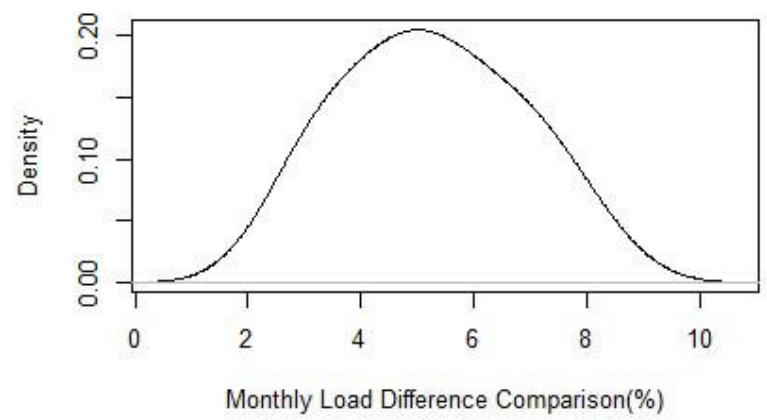

11

12
Figure 11: Statistics for monthly load differences between implementing the EVNS and shared combinations (both are compared to baseline control) in the office building

It can be seen that a minimum of $10.4 \%$ and a maximum of $28.3 \%$ monthly load reduction were achieved by the EVNS (boxplot in Figure 11). Since Los Angeles is in a cooling-dominant climate zone, more load 
reduction was expected during the warm seasons than the cold seasons. During the summer vacation (from mid-May to Mid-August) and winter recess (from mid-December to mid-January), more loads were reduced as occupied periods were generally shortened, especially for the hot days when more cooling was required to maintain the desired indoor thermal conditions while the spaces were not always occupied. Load reduction resulting from EVNS had the similar trend (correlation plot in Figure 11) with that of implementing the shared combination of setpoint/setback schedules and distances (selected from 64 combinations for each month) for zones but was greater than the reduction from shared combination (plot of monthly load difference in Figure 11). The difference is approximately normally distributed (kernel density in Figure 11). This finding demonstrates the EVNS is effective to search combinations that could quantify the relationship between occupancy transitions and heating/cooling loads at the building level. Next, the zones on the third floor were assigned with random solutions of setpoint/setback schedules and distances and the loads were compared with the loads from implementing the EVNS for the entire year. This process was repeated for 100 times and the corresponding loads were all greater than the loads from the one received by implementing the EVNS (Figure 12). The setpoint/setback schedules and distances selected by the EVNS for all individual zones minimized heating/cooling loads based on occupancy transitions, similar to the findings in Section 4.

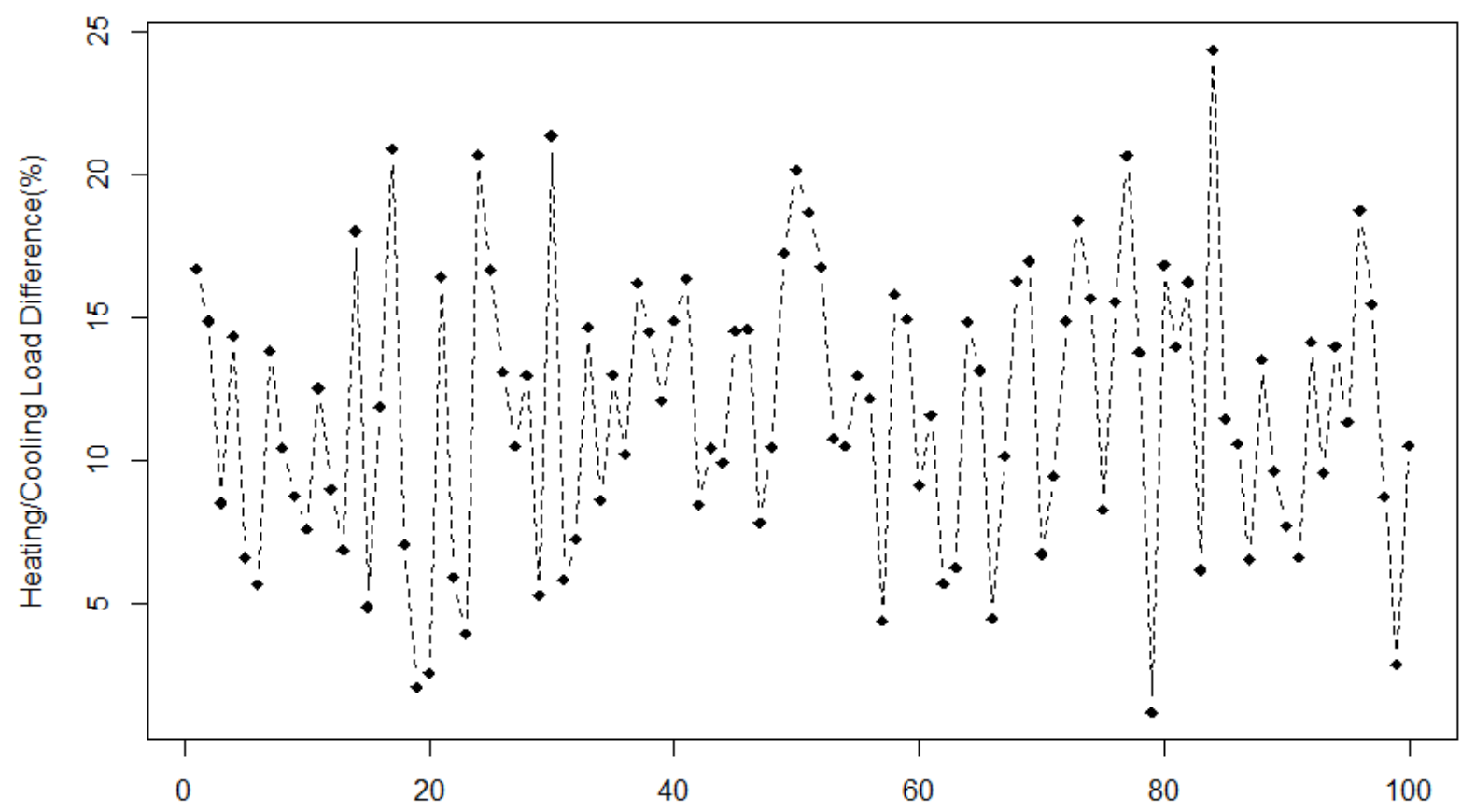

Number of random solutions

Figure 12: Load differences between random solutions and calculated global optimum (denoted as the line: $\mathrm{Y}=0)$

\section{Conclusions}

In this paper, we introduced a data-driven approach using an enhanced variable neighborhood search algorithm to determine setpoint/setback schedules and distances for all individual zones in a building 
based on occupancy transitions, by which heating/cooling loads could be minimized. Given certain occupancy data and weather, the difference in the loads resulting from the proposed approach and the baseline control shows the quantitative relationship between occupancy transitions and heating/cooling loads for a certain period of time. A small size reference building was used to validate the performance of integrating variable neighborhood search with a heuristic update for finding the optimal solution. It was demonstrated that the convergence of the search was not influenced by different occupancy assignments or initial solutions, and there was no random solution that could outperform the proposed approach to reduce the heating/cooling loads based on occupancy transitions at the building level. A real-world office building was also simulated as a case study for introducing how to apply the EVNS to real-world occupancy transitions-loads analysis. A minimum of $10.4 \%$ and a maximum of $28.3 \%$ load reduction were achieved compared to the baseline control by setting different zones with optimized combinations of setpoint/setback schedules and distances. To the best of our knowledge, the proposed approach is the first investigation in literature to provide a general way of quantifying the relationships between occupancy state transitions and heating/cooling loads at the building level for any given period of time. The approach takes into consideration the synergies among adjacent zones, which result in load transitions and balance by determining optimal setpoint/setback schedules and distances for individual zones. In the implementation and validation of the approach, we made assumptions related to comfort ranges in the simulated buildings as personalized thermal comfort approaches were not in the scope of our explorations. However, any comfort approach could be used and the resulting comfort information could be an input to our approach, replacing the comfort assumptions made in this paper. The approach in this paper is based on the analysis of historical data therefore cannot dynamically generate real-time solutions. However, it integrates the patterns of real-time occupancy state transitions and provides a robust control over time which is important for occupant comfort and system stability. The calculated setpoint/setback schedules and distances could be used as initial settings to adapt to the changes of occupancy state transitions and finally converge to optimal setpoint/setback combinations. Moreover, it can be applied to other buildings with different HVAC systems.

27 It is important to note that, in this paper, setback was set to be higher than the setpoint, as cooling is 28 dominant and heating is seldom provided in Los Angeles. Yet, this assumption does not influence the way the algorithm works, and the EVNS could also be used in the heating-dominant areas as well as in the heating/cooling mixed areas. Several improvement areas are noted and outlined here for future explorations. We will test the EVNS in different climate zones for analyzing the patterns of heating loads and cooling loads separately. Different types of commercial buildings will be used to explore the correlations among a building's physical characteristics, heating/cooling loads, and occupancy transitions. The relationships between occupancy transitions and heating/cooling are time-variant, which have to be calculated for different lengths of periods (e.g., one year, one month, or one week). Although the number of simulation runs is significantly reduced, hundreds of iterations still take considerable amount of time. In our future work, we will use statistical analysis to find the relationships and patterns among the different levels of occupancy transitions, weather, and optimized combinations of setpoint/setback schedules and distances. Factors including the arrival time, departure time, morning/afternoon average absence length, break lengths, which are possible to influence the occupancy transitions-loads relationships, will also be identified by computer learning. Lastly, in this paper the setpoint was not restored until the space became occupied again, as occupancy prediction has not been incorporated to the approach. Since additional errors might be introduced when prediction is used to recondition a space from 
setback to setpoint before occupants actually occupy their spaces, the energy implications of integrating pre-control strategies will be systematically analyzed in our future studies to determine the dynamic time point of switching from setback period to reconditioning period. Online learning for integrating occupancy state transitions and real-time setpoint/setback control will be investigated in future.

\section{Acknowledgements}

This material is based upon the work supported by the National Science Foundation under Grant No. 1351701. Any opinions, findings, and conclusions or recommendations expressed in this material are those of the author(s) and do not necessarily reflect the views of the National Science Foundation. The occupancy data and building performance data were collected under Grant No. DE-EE0004019. Computation for the work described in this paper was partly supported by the University of Southern California's Center for High-Performance Computing (hpc.usc.edu).

\section{References}

[1] U.S. Department of Energy. Better Buildings, Brighter Future. 2013.

[2] U.S. Department of Energy. Building Energy Data Book. Available at: http://buildingsdatabook.eren.doe.gov/ChapterIntro3.aspx. Accessed on: 05/01, 2016.

[3] U.S. Department of Energy. Building Energy Consumption and Efficiency- Commercial Building Energy Consumption Survey. Available at: http://www.eia.gov/consumption/commercial/. Accessed on: 04/20, 2016.

[4] Carbon Trust. Building Controls- Realising Savings through the Use of Controls. 2012.

[5] Roetzel A, Tsangrassoulis A, Dietrich U. Impact of Building Design and Occupancy on Office

Comfort and Energy Performance in Different Climates. Build.Environ. 2014. 71: 165-75.

[6] Erickson VL, Carreira-Perpiñán MÁ, Cerpa AE. Occupancy Modeling and Prediction for Building energy management. ACM Transactions on Sensor Networks (TOSN). 2014. 10.(3):42.

[7] Martani C, Lee D, Robinson P, Britter R, Ratti C. ENERNET: Studying the Dynamic Relationship between Building Occupancy and Energy Consumption. Energy Build. 2012. 47: 584-91.

[8] Gul MS, Patidar S. Understanding the Energy Consumption and Occupancy of A Multi-Purpose Academic Building. Energy Build. 2015. 87: 155-65.

[9] Pritoni M, Meier A, and Perry D. Human Factors in Climate Controls for Small Commercial Buildings. Johnson Control Technical Report. 2011.

[10] Yang Z, Becerik-Gerber B. How Does Building Occupancy Influence Energy Efficiency of HVAC Systems. The Applied Energy Symposium on Low-Carbon Cities and Urban Energy Systems. 2015. [11] Yang Z, Ghahramani A, Becerik-Gerber B. Effects of Variant Occupancy Transitions on the Energy Implications of Setpoint/Setback Control Policies. Symposium on Sustainable Human-Building Ecosystems. 2015: 90-98.

[12] Lo LJ, Novoselac A. Localized Air-Conditioning with Occupancy Control in An Open Office. Energy Build. 2010. 42 (7):1120-8.

[13] Zhu Y, Liu M, Batten T, Noboa H, Claridge DE, and Turner WD. Optimization of Control Strategies for HVAC Terminal Boxes. The 12th Symposium on Improving Building Systems in Hot and Humid Climates.2000.

[14] Henze GP, Felsmann C, Knabe G. Evaluation of Optimal Control for Active and Passive Building Thermal Storage. International Journal of Thermal Sciences. 2004. 43 (2):173-83.

[15] Deng K, Barooah P, Mehta PG, and Meyn SP. Building Thermal Model Reduction via Aggregation of States. The IEEE American Control Conference (ACC). 2010. 
[16] Oldewurtel F, Parisio A, Jones CN, and Morari M. Energy Efficient Building Climate Control Using Stochastic Model Predictive Control and Weather Predictions. The IEEE American Control Conference (ACC). 2010.

[17] Aswani A, Master N, Taneja J, Culler D, and Tomlin C. Reducing Transient and Steady State Electricity Consumption in HVAC Using Learning-Based Model-Predictive Control. Proceedings of IEEE. 2012. 100 (1): 240-53.

[18] Gao G, Whitehouse K. The Self-Programming Thermostat: Optimizing Setback Schedules Based on Home Occupancy Patterns. The 1st ACM Workshop on Embedded Sensing Systems for EnergyEfficiency in Buildings. 2009.

[19] Dong B, Lam KP, and Neuman C. Integrated Building Control Based on Occupant Behavior Pattern Detection and Local Weather Forecasting. The 12th International Building Performance Simulation Association Conference. 2011.

[20] NEST Lab. Life with Nest Thermostat. Available at: https://nest.com/thermostat/inside-andout/\#nest-sense. Accessed on: 05/01, 2016.

[21] Lu J, Sookoor T, Srinivasan V, et al. The Smart Thermostat: Using Occupancy Sensors to Save Energy in Homes. The 8th ACM Conference on Embedded Networked Sensor Systems. 2010.

[22] Telkonet SmartEnergy. Available at: http://www.telkonet.com/index.php. Accessed on: 05/01, 2016. [23] Erickson VL, Carreira-Perpiñán MÁ, and Cerpa AE. OBSERVE: Occupancy-Based System for Efficient Reduction of HVAC Energy. The 10th International Conference on Sensor Networks (IPSN). 2011.

[24] Agarwal Y, Balaji B, Gupta R, Lyles J, Wei M, and Weng T. Occupancy-Driven Energy

Management for Smart Building Automation. The 2nd ACM Workshop on Embedded Sensing Systems for Energy-Efficiency in Building. 2010.

[25] Johnson Controls. Personal environments. 2010. Available at:

http://johnsoncontrols.ae/content/in/en/products/building_efficiency/integrated_hvac_systems/hvac/perso nal_environments.html. Accessed on: 05/01. 2016

[26] Sookoor T, Whitehouse K. RoomZoner: Occupancy-Based Room-Level Zoning of A Centralized HVAC System. The ACM/IEEE 4th International Conference on Cyber-Physical Systems. 2013. [27] Chandan V, Alleyne AG. Optimal Control Architecture Selection for Thermal Control of Buildings. The IEEE American Control Conference (ACC). 2011.

[28] Oldewurtel F, Sturzenegger D, Morari M. Importance of Occupancy Information for Building Climate Control. Applied Energy. 2013. 101:521-32.

[29] Goyal S, Ingley HA and Barooah P. Effect of Various Uncertainties on the Performance of Occupancy-Based Optimal Control of HVAC Zones. The IEEE 51st Annual Conference on Decision and Control (CDC). 2012.

[30] Ghahramani A, Zhang K, Dutta K, Yang Z, Becerik-Gerber B. Energy Savings from Temperature Setpoints and Deadband: Quantifying the Influence of Building and System Properties on Savings. Appl.Energy. 2016. 165: 930-42.

[31] Hoyt T, Arens E, Zhang H. Extending Air Temperature Setpoints: Simulated Energy Savings and Design Considerations for New and Retrofit Buildings. Build.Environ. 2015. 88: 89-96.

[32] Blight TS, Coley DA. Sensitivity Analysis of the Effect of Occupant Behaviour on the Energy Consumption of Passive House Dwellings. Energy Build. 2013. 66: 183-92.

[33] Wei S, Jones R, de Wilde P. Driving Factors for Occupant-Controlled Space Heating in Residential Buildings. Energy Build. 2014. 70: 36-44.

[34] Karjalainen S. Should We Design Buildings That are Less Sensitive to Occupant Behaviour? A Simulation Study of Effects of Behaviour and Design on Office Energy Consumption. Energy Efficiency. 2016:1-14.

[35] Balaji B, Xu J, Nwokafor A, Gupta R and Agarwal Y. Sentinel: Occupancy based HVAC Actuation Using Existing WiFi Infrastructure within Commercial Buildings. Proceedings of the 11th ACM Conference on Embedded Networked Sensor Systems. 2013. 
1 [36] Beltran A, Erickson VL and Cerpa AE. Thermosense: Occupancy Thermal based sSensing for HVAC Control. Proceedings of the 5th ACM Workshop on Embedded Systems For Energy-Efficient Buildings. 2013.

[37] Milenkovic M and Amft O. An Opportunistic Activity-Sensing Approach to Save Energy in Office Buildings. Proceedings of the fourth international conference on Future energy systems. 2013. [38] Terrill TJ, Rasmussen BP. An Evaluation of HVAC Energy Usage and Occupant Comfort in Religious Facilities. Energy Build. 2016. 128: 224-35.

[39] Goyal S, Ingley HA, Barooah P. Occupancy-based Zone-Climate Control for Energy-Efficient Buildings: Complexity vs. Performance. Appl.Energy. 2013. 106: 209-21.

[40] Ioannou A and Itard L. Energy Performance and Comfort in Residential Buildings: Sensitivity for Building Parameters and Occupancy. Energy Build. 2015. 92: 216-33.

[41] Korolija I, Marjanovic-Halburd L, Zhang Y, and Hanby VI. Influence of Building Parameters and HVAC Systems Coupling on Building Energy Performance. Energy Build. 2011.43 (6):1247-53.

[42] Martani C, Lee D, Robinson P, Britter R, and Ratti C. ENERNET: Studying the Dynamic Relationship between Building Occupancy and Energy Consumption. Energy Build. 2012. 47:584-91. [43] Russell SJ, Norvig P, Canny JF, Malik JM, Edwards DD. Artificial Intelligence: A Modern Approach. Prentice Hall Upper Saddle River. 2003.

[44] Banzhaf W, Nordin P, Keller RE, Francone FD. Genetic Programming: An Introduction. Morgan Kaufmann Publishers San Francisco. 1998.

[45] Brooks SP, Morgan BJ. Optimization Using Simulated Annealing. The Statistician. 1995. 241-57. [46] Glover F, Laguna M. Tabu Search. Springer. 2013.

[47] Deru M, Field K, Studer D, Benne K, Griffith B, Torcellini P, et al. U.S. Department of Energy Commercial Reference Building Models of the National Building Stock. NREL Technical Report. 2011. [48] Yang Z and Becerik-Gerber B. Cross-Space Building Occupancy Modeling by Contextual Information Based Learning. The 2nd ACM International Conference on Embedded Systems for EnergyEfficient Built Environments. 2015.

[49] American Society of Heating, Refrigerating and Air-Conditioning Engineers. ANSI/ASHRAE

28 Standard 62.1-2010: Ventilation for Acceptable Indoor Air Quality. 2010. 